\title{
Some Generalizations of Weibull Distribution and Related Processes
}

\author{
K. Jayakumar \\ Department of Statistics, University of Calicut \\ Calicut University P.O. - 673 635, Kerala, India \\ jkumar19@rediffmail.com \\ M. Girish Babu \\ Department of Statistics, Govt. Arts \& Science College \\ Meenchanda, Calicut-673 018, Kerala, India \\ giristat@gmail.com
}

Received 31 December 2014

Accepted 6 August 2015

\begin{abstract}
A new class of distributions containing Marshall-Olkin extended Weibull distribution is introduced. The role of this distribution in the study of minification process is established. A new class of distributions that includes the Laplace and Logistic distributions is introduced. Properties and generation of Marshall-Olkin extended Double Weibull distribution and Marshall-Olkin extended asymmetric double Weibull distribution are discussed. The distribution of daily stock price index of Bombay stock exchange (INDIA) is identified as generalised asymmetric double Weibull model and its estimation and predictions are done.

Keywords: Autoregressive process; Double Weibull distribution; Generalized asymmetric double Weibull distribution; Marshall-Olkin extended Weibull distribution; Minification process; Stationarity.
\end{abstract}

2000 Mathematics Subject Classification: 62M10, 60E05, 60G10

\section{Introduction}

The Weibull family of distributions has been widely used in the analysis of survival data especially in medical and engineering applications. This family is suitable in situations where the hazard rate is constant or monotone. Many parametric families have been considered for modelling survival data with a more general shape for the hazard rate. The Weibull distribution which includes the Exponential and Rayleigh distributions as special cases have a wide variety of applications in physical science, life science and reliability analysis, see N.L. Johnson et al. [1].

For the last two decades several new models have been proposed that are either in derived form or in some way related to the Weibull distribution. They are suitable to model complex data sets. For modelling monotone hazard rates, the Weibull distribution may be an initial choice because of its negatively and positively skewed density shapes. However, this distribution does not provide a reasonable parametric fit for modelling phenomenon with non-monotone failure rates such as the bathtub shaped and the unimodal failure rates which are common in reliability and biological studies. Such bathtub hazard curves have nearly flat middle portions and the corresponding densities have a positive anti mode. Human mortality with a high infant mortality rate which reduces rapidly to reach a low rate is an example for bathtub shaped failure rate. 
Unimodal failure rates can be observed in course of a disease whose mortality reaches a peak after some finite period and then declines gradually.

The three parameter Weibull distribution is given by the distribution function

$$
F(x, \theta)=1-\exp \left[-\left(\frac{x-\tau}{\alpha}\right)^{\beta}\right], x \geq \tau
$$

The parameters of the distribution are given by the set $\theta=\{\alpha, \beta, \tau\}$ with $\alpha>0, \beta>0$ and $\tau \geq 0$. The parameters $\alpha, \beta$ and $\tau$ are the scale, shape and location parameters of the distribution respectively. The distribution is named after Waloddi Weibull who was the first to promote the usefulness of this model to explain the strength of materials, D.N.P. Murthy et al. [2].

The need for extended form of the Weibull distribution arises in many applied areas. A.W. Marshall, I. Olkin [3] proposed a method of adding a new parameter to an existing distribution. The resulting distribution is called the Marshall-Olkin extended distribution, includes the original distribution as a special case and gives more flexibility to model various types of data. Let $\bar{F}(x)=1-F(x)$ denote the baseline survivor function of a continuous random variable $\mathrm{X}$ which depends on a parameter $\beta=\left(\beta_{1}, \beta_{2}, \ldots, \beta_{q}\right)^{\prime}$ of dimension $q$. The Marshall Olkin extended distribution has survival function given by,

$$
\bar{G}(x)=\frac{\alpha \bar{F}(x)}{1-\bar{\alpha} \bar{F}(x)}=\frac{\alpha \bar{F}(x)}{F(x)+\alpha \bar{F}(x)}, \quad-\infty<x<\infty, \alpha>0
$$

where $\bar{\alpha}=1-\alpha$. For $\alpha=1, \bar{G}(x)=\bar{F}(x)$.

The particular case that $\mathrm{F}$ is an exponential distribution yields a two-parameter family of distributions which may sometimes be a competitor to the Weibull and Gamma families. The corresponding extended exponential family was discussed in [3].

The probability density function (pdf) of $\mathrm{G}(\mathrm{x})$ is given by

$$
g(x)=\frac{\alpha f(x)}{[1-\bar{\alpha} \bar{F}(x)]^{2}}, \quad-\infty<x<\infty
$$

The hazard rate is

$$
h(x ; \alpha)=\frac{1}{[1-\bar{\alpha} \bar{F}(x)]} \cdot h_{F}(x) \quad,-\infty<x<\infty
$$

where $h_{F}(x)$ denote the hazard rate function of the original model with distribution function $F$.The properties of this distribution are studied in [3].

The distribution function and density function of the standard Weibull distribution with parameter $\beta>0$ is given by,

$$
F(x)=1-e^{-x^{\beta}}, \quad x>0
$$

and

$$
f(x)=\beta x^{\beta-1} e^{-x^{\beta}}, \quad x>0
$$

Then the cumulative distribution function $\mathrm{G}(\mathrm{x})$ of the Marshall-Olkin Extended Weibull (MOEW) distribution is given by,

$$
G(x)=\frac{1-e^{-x^{\beta}}}{1-\bar{\alpha} e^{-x^{\beta}}}, \quad x>0, \quad \beta>0
$$

The survival function is

$$
\bar{G}(x)=\frac{1}{1+\frac{1}{\alpha}\left(e^{x^{\beta}}-1\right)}
$$

We denote the distribution with survival function (1.8) as $\operatorname{MOEW}(\alpha, \beta)$. 
Models with minification structures have been introduced in the literature as an alternative to the nonGaussian time series models where the generating functions usually used as a main tool for their analysis do not have closed form expressions. The study on minification process began with the work of L.V. Tavares [4]. He introduced an autoregressive minification process

$$
X_{n}= \begin{cases}X_{0}, & n=0 \\ k \min \left(X_{n-1}, \varepsilon_{n}\right), & n \geq 1\end{cases}
$$

where $k>1$ is a constant and $\left\{\varepsilon_{n}\right\}$ is an innovation process of i.i.d random variables such that $\left\{X_{n}\right\}$ is a stationary Markov process. He considered a particular case where $\left\{\varepsilon_{n}\right\}$ is a sequence of i.i.d. exponential random variables with mean $\theta(k-1)$ and $X_{0}$ is exponential with mean $\theta$. This model generates a first order autoregressive exponential process with mean $\theta$ and is useful in hydrological applications.

C.H. Sim [5] developed a first order autoregressive Weibull process. He showed that $\left\{X_{n}\right\}$ in equation (1.9) are stationary Weibull random variables with survival function $e^{\left\{\frac{-\theta X^{c}}{k^{C}-1}\right\}}$ if and only if $\left\{\varepsilon_{n}\right\}$ is a sequence of i.i.d. Weibull random variables with survival function $e^{-\theta X^{c}}$.

K.K. Jose et al. [6] developed different types of autoregressive processes with minification structure and max-min structure. K.K. Jose [7] considered various Marshall-Olkin distributions and developed autoregressive minification processes with stationary marginals as exponential, Weibull, uniform, Pareto, Gumbel etc.

In section 2, we discuss the MOEW Minification process. A new class of distribution containing MOEW distribution is introduced. The role of this distribution in the study of minification process is established. Properties and generation of Marshall-Olkin extended Double Weibull distribution and Marshall-Olkin extended asymmetric double Weibull distribution are discussed in section 3. In section 4, the distribution of daily stock price index of Bombay stock exchange (INDIA) is identified as generalised asymmetric double Weibull model and its estimation and predictions are done.

\section{Marshall-Olkin Extended Weibull Minification Process}

Here we develop an autoregressive minification process of order one with Weibull distribution as marginal. We have the following theorems.

Theorem 2.1. The process

$$
X_{n}=\left\{\begin{array}{cl}
\varepsilon_{n} & \text { with probability } \gamma \\
\min \left(X_{n-1}, \varepsilon_{n}\right) & \text { with probability } 1-\gamma
\end{array}\right.
$$

where $0<\gamma<1$, defines a stationary $\mathrm{AR}(1)$ minification process if and only if $\varepsilon_{n}$ 's are i.i.d. MOEW with $X_{0} \stackrel{d}{=}$ Weibull

Proof. The model (2.1) in terms of survival function is

$$
P\left(X_{n}>x\right)=P\left(\varepsilon_{n}>x\right)\left[\gamma+(1-\gamma) P\left(X_{n-1}>x\right)\right]
$$

That is,

$$
\bar{F}_{X_{n}}(x)=\bar{F}_{\varepsilon_{n}}(x)\left[\gamma+(1-\gamma) \bar{F}_{X_{n-1}}(x)\right]
$$

If $\left\{X_{n}\right\}$ is stationary with Weibull marginals, then

$$
\bar{F}_{\varepsilon_{n}}(x)=\frac{\bar{F}_{X}(x)}{\gamma+(1-\gamma) \bar{F}_{X}(x)}=\frac{e^{-x^{\beta}}}{\gamma+(1-\gamma) e^{-x^{\beta}}}=\frac{1}{1+\gamma\left(e^{x^{\beta}}-1\right)}
$$

That is , $\varepsilon_{n}$ 's are extended Weibull. 
Conversely, if $\varepsilon_{n}$ 's are extended Weibull with $X_{0} \underline{\underline{d}}$ Weibull, then $\left\{X_{n}\right\}$ defines a stationary process. From (2.2),

$$
\bar{F}_{X_{1}}(x)=\bar{F}_{\varepsilon_{1}}(x)\left[\gamma+(1-\gamma) \bar{F}_{X_{0}}(x)\right]=\frac{1}{1+\gamma\left(e^{x^{\beta}}-1\right)}\left[\gamma+(1-\gamma) e^{-x^{\beta}}\right]=e^{-x^{\beta}} .
$$

That is, $X_{1} \stackrel{d}{=}$ Weibull.

If we assume that $X_{n-1} \stackrel{d}{=}$ Weibull, we get $X_{n} \underline{\underline{d}}$ Weibull. Hence the process $\left\{X_{n}\right\}$ is stationary with Weibull marginals.

Theorem 2.2. The AR(1) minification process

$$
X_{n}=\left\{\begin{array}{cl}
\varepsilon_{n} & \text { with probability } \delta \\
\min \left(X_{n-1}, \varepsilon_{n}\right) & \text { with probability } 1-\delta
\end{array}\right.
$$

where $0<\delta<1$, defines a stationary $\mathrm{AR}(1)$ minification process with extended Weibull marginals if and only if $\varepsilon_{n}$ 's are $\operatorname{MOEW}\left(\frac{\alpha}{\delta}, \beta\right)$ and $X_{0} \stackrel{d}{=} \operatorname{MOEW}(\alpha, \beta)$.

Proof. Equation (2.3) in terms of survival function is

$$
P\left(X_{n}>x\right)=P\left(\varepsilon_{n}>x\right)\left[\delta+(1-\delta) P\left(X_{n-1}>x\right)\right]
$$

That is

$$
\bar{F}_{X_{n}}(x)=\bar{F}_{\varepsilon_{n}}(x)\left[\delta+(1-\delta) \bar{F}_{X_{n-1}}(x)\right]
$$

If $\left\{X_{n}\right\}$ is stationary, then

$$
\bar{F}_{\varepsilon_{n}}(x)=\frac{\bar{F}_{X}(x)}{\delta+(1-\delta) \bar{F}_{X}(x)}=\frac{\frac{\alpha e^{-x^{\beta}}}{\left(1-\bar{\alpha} e^{-x^{\beta}}\right)}}{\delta+(1-\delta)\left(\frac{\alpha e^{-x^{\beta}}}{\left(1-\bar{\alpha} e^{-x^{\beta}}\right)}\right)}=\frac{1}{1+\frac{\delta}{\alpha}\left(e^{x^{\beta}}-1\right)},
$$

which is extended Weibull.

Conversely, if $\varepsilon_{n}$ 's are $\operatorname{MOEW}\left(\frac{\alpha}{\delta}, \beta\right)$ and $X_{0} \underline{\underline{d}} \operatorname{MOEW}(\alpha, \beta)$, then from (2.4),

$$
\bar{F}_{X_{1}}(x)=\bar{F}_{\varepsilon_{1}}(x)\left[\delta+(1-\delta) \bar{F}_{X_{0}}(x)\right]=\frac{1}{1+\frac{\delta}{\alpha}\left(e^{x^{\beta}}-1\right)}\left[\delta+(1-\delta) \frac{1}{1+\frac{1}{\alpha}\left(e^{x^{\beta}}-1\right)}\right]=\frac{1}{1+\frac{1}{\alpha}\left(e^{x^{\beta}}-1\right)} .
$$

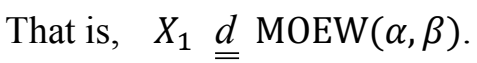

If $X_{n-1} \stackrel{d}{=} \operatorname{MOEW}(\alpha, \beta)$, we get $X_{n} \stackrel{d}{\underline{d}} \operatorname{MOEW}(\alpha, \beta)$. Hence the process $\left\{X_{n}\right\}$ is stationary with $\operatorname{MOEW}(\alpha, \beta)$ marginals. Based on this, we define the first order MOEW autoregressive minification process as follows: Let $X_{0} \underline{\underline{d}} \operatorname{MOEW}(\alpha, \beta)$ and for $n=1,2,3, \ldots$, define

$$
X_{n}=\left\{\begin{array}{cl}
\varepsilon_{n} & \text { with probability } \delta \\
\min \left(X_{n-1}, \varepsilon_{n}\right) & \text { with probability } 1-\delta
\end{array}\right.
$$

where $0<\delta<1$ and $\left\{\varepsilon_{n}\right\}$ is a sequence of i.i.d. $\operatorname{MOEW}\left(\frac{\alpha}{\delta}, \beta\right)$ random variables.

Now we define a new class of distribution containing MOEW distribution. Consider the class of distributions with survival function

where $\psi(x)$ satisfies the functional equation

$$
\bar{G}(x)=\frac{\alpha}{e^{\psi(x)}-\bar{\alpha}}, \quad \alpha>0, x>0
$$




$$
\psi(x)=\frac{1}{p} \psi\left(p^{\frac{1}{\beta}} x\right), \quad \beta>0, \quad 0<p<1
$$

We denote the distribution with survival function (2.5) as Marshall - Olkin extended semi-Weibull $\operatorname{MOESW}(\alpha, \beta, p)$.

Solution of (2.6) is $\psi(x)=x^{\beta} h(x)$, where $h(x)=h\left(p^{\frac{1}{\beta}} x\right)$ (for proof see A.M. Kagan et al.[8], p.163). As an example of $\mathrm{h}(\mathrm{x})$ function, we have $h_{1}(x)=\exp \{r \operatorname{Cos}(\beta \ln x)\}$. Then $h(x)$ satisfies (2.6) with $p=$ $e^{-2 \pi}$.

Theorem 2.3. The AR(1) minification process

$$
X_{n}=\left\{\begin{array}{cl}
\varepsilon_{n} & \text { with probability } v \\
\min \left(X_{n-1}, \varepsilon_{n}\right) & \text { with probability } 1-v
\end{array}\right.
$$

where $0<v<1$, defines a stationary $\mathrm{AR}(1)$ minification process with Marshall-Olkin extended semiWeibull marginals if and only if $\varepsilon_{n}$ 's are i.i.d Marshall-Olkin extended semi-Weibull with $X_{0} \underline{\underline{d}}$ extended semi-Weibull.

Proof. Stationarity (2.7) in terms of Survival function becomes,

$$
\bar{F}_{\varepsilon_{n}}(x)=\frac{\bar{F}_{X}(x)}{v+(1-v) \bar{F}_{X}(x)}=\frac{\frac{\alpha}{\left(e^{\psi(x)}-\bar{\alpha}\right)}}{v+(1-v)\left(\frac{\alpha}{\left(e^{\psi(x)}-\bar{\alpha}\right)}\right)}=\frac{1}{1+\frac{v}{\alpha}\left(e^{\psi(x)}-1\right)},
$$

which is extended semi-Weibull.

Conversely, if $\varepsilon_{n}$ 's are $\operatorname{MOESW}(\alpha, \beta, p)$ and $X_{0} \underline{\underline{d}} \operatorname{MOESW}(\alpha, \beta, p)$, then from (2.7),

$$
\bar{F}_{X_{1}}(x)=\bar{F}_{\varepsilon_{1}}(x)\left[v+(1-v) \bar{F}_{X_{0}}(x)\right]=\frac{1}{1+\frac{v}{\alpha}\left(e^{\psi(x)}-1\right)}\left[v+(1-v) \frac{1}{1+\frac{1}{\alpha}\left(e^{\psi(x)}-1\right)}\right]=\frac{1}{1+\frac{1}{\alpha}\left(e^{x^{\psi(x)}}-1\right)} .
$$

That is, $X_{1} \underline{\underline{d}} \operatorname{MOESW}(\alpha, \beta, p)$. If $X_{n-1} \stackrel{d}{=} \operatorname{MOESW}(\alpha, \beta, p)$, we get $X_{n} \stackrel{d}{=} \operatorname{MOESW}(\alpha, \beta, p)$. Hence the

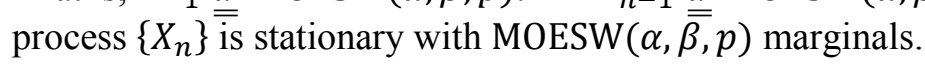

\section{Marshall-Olkin Extended Double Weibull distribution}

The distribution function of a double Weibull distribution is given by

$$
F(x ; \alpha, \beta, \tau)=\left\{\begin{array}{c}
\frac{1}{2} \exp \left\{-\left[\frac{\tau-x}{\alpha}\right]^{\beta}\right\} \text { for } x<\tau \\
1-\frac{1}{2} \exp \left\{-\left[\frac{x-\tau}{\alpha}\right]^{\beta}\right\} \text { for } x \geq \tau
\end{array}\right.
$$

For $x \geq \tau$ the distribution is similar to the three parameter Weibull distribution and for $x<\tau$ it is similar to the reflected Weibull distribution. For double Weibull distribution the support is $-\infty<x<\infty$.

N. Balakrishnan, S. Kocherlakota [9] introduced a double Weibull distribution and its properties concerning order statistics are discussed. They studied a special case of (3.1) where $\alpha=1$ and $\tau=0$. In this case the density function is given by

$$
f(x)=\frac{\beta}{2}|x|^{\beta-1} \exp \left(-|x|^{\beta}\right), \quad-\infty<x<\infty, \quad \beta>0
$$

A real valued extension of the Marshall-Olkin Weibull distribution is possible and the corresponding probability density function is given by $g(x)=\frac{1}{2} f(|x|)$. The random variable $\mathrm{X}$ with this probability density 
function is termed as Generalized Symmetric Double Weibull (GSDW) random variable and the corresponding probability density function with the location and scale parameters included is given by

$$
g(x)=\frac{\alpha \beta}{2 \sigma^{\beta}} \frac{|x-\mu|^{\beta-1} e^{\left|\frac{x-\mu}{\sigma}\right|^{\beta}}}{\left(e^{\left|\frac{x-\mu}{\sigma}\right|^{\beta}}-\bar{\alpha}\right)^{2}}, \quad-\infty<x, \mu<\infty, 0<\beta, \alpha<\infty, \sigma>0
$$

Then $X$ is said to follow generalized symmetric double Weibull (GSDW) distribution with parameters $\alpha, \beta, \mu$ and $\sigma$ and we write it as $X \underline{\underline{d}} \operatorname{GSDW}(\alpha, \sigma, \beta, \mu)$

For $\beta=1$ and $\alpha=1$ the distribution reduces to Laplace distribution and for $\beta=1$ and $\alpha=2$ it becomes the Logistic distribution. Therefore the GSDW distribution can be viewed as a generalization to Laplace as well as to Logistic distribution.

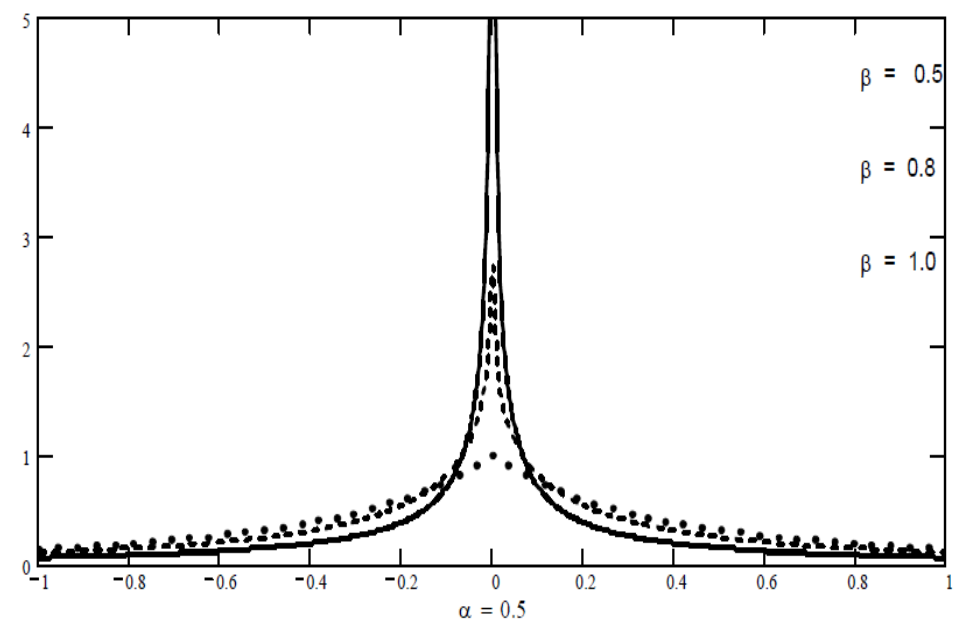

Fig.1. Density plot of generalized symmetric double Weibull distribution

The distribution function of $\operatorname{GSDW}(\alpha, \sigma, \beta, \mu)$ is given by

$$
F(x)= \begin{cases}\frac{\alpha}{2} \frac{1}{\left(e^{\left(\frac{-(x-\mu)}{\sigma}\right)^{\beta}}-\bar{\alpha}\right)} & \text { if } \mathrm{x} \leq 0 \\ 1-\frac{\alpha}{2} \frac{1}{\left(e^{\left(\frac{(x-\mu)}{\sigma}\right)^{\beta}}-\bar{\alpha}\right)} & \text { if } \mathrm{x} \geq 0\end{cases}
$$

For different values of $\alpha$ and $\beta$, the cumulative probabilities of the generalized symmetric double Weibull (GSDW) is computed by taking $\mu=0, \sigma=1$ and displaced in the table below. 
Table 1.Cumulative Probabilities of GSDW distribution

\begin{tabular}{|c|c|c|c|c|c|c|c|c|}
\hline \multirow{2}{*}{$x$} & \multicolumn{2}{|c|}{$\alpha=0.5$} & \multicolumn{2}{|c|}{$\alpha=1.0$} & \multicolumn{2}{|c|}{$\alpha=1.5$} & \multicolumn{2}{|c|}{$\alpha=2.0$} \\
\hline & $\beta=0.5$ & $\beta=1.0$ & $\beta=0.5$ & $\beta=1.0$ & $\beta=0.5$ & $\beta=1.0$ & $\beta=0.5$ & $\beta=1.0$ \\
\hline 0 & 0.5 & 0.5 & 0.5 & 0.5 & 0.5 & 0.5 & 0.5 & 0.5 \\
\hline 1 & 0.8873 & 0.8873 & 0.81606 & 0.81606 & 0.76696 & 0.76696 & 0.73106 & 0.73106 \\
\hline 2 & 0.93081 & 0.96371 & 0.87844 & 0.93233 & 0.83742 & 0.90493 & 0.80443 & 0.8808 \\
\hline 3 & 0.95148 & 0.98724 & 0.91154 & 0.97511 & 0.87809 & 0.96357 & 0.84967 & 0.95257 \\
\hline 4 & 0.96371 & 0.99538 & 0.93233 & 0.99084 & 0.90493 & 0.98639 & 0.8808 & 0.98201 \\
\hline 5 & 0.97177 & 0.99831 & 0.94656 & 0.99663 & 0.92391 & 0.99496 & 0.90344 & 0.99331 \\
\hline 6 & 0.97744 & 0.99938 & 0.95683 & 0.99876 & 0.93793 & 0.99814 & 0.92052 & 0.99753 \\
\hline 7 & 0.98161 & 0.99977 & 0.96452 & 0.99954 & 0.94861 & 0.99932 & 0.93375 & 0.99909 \\
\hline 8 & 0.98477 & 0.99992 & 0.97045 & 0.99983 & 0.95694 & 0.99975 & 0.94419 & 0.99966 \\
\hline 9 & 0.98724 & 0.99997 & 0.97511 & 0.99994 & 0.96357 & 0.99991 & 0.95257 & 0.99988 \\
\hline 10 & 0.98919 & 0.99999 & 0.97884 & 0.99998 & 0.96891 & 0.99997 & 0.95939 & 0.99995 \\
\hline 11 & 0.99076 & 1 & 0.98186 & 0.99999 & 0.97328 & 0.99999 & 0.96499 & 0.99998 \\
\hline 12 & 0.99205 & 1 & 0.98435 & 1 & 0.97689 & 1 & 0.96965 & 0.99999 \\
\hline 13 & 0.99311 & 1 & 0.98641 & 1 & 0.97989 & 1 & 0.97355 & 1 \\
\hline 14 & 0.994 & 1 & 0.98814 & 1 & 0.98242 & 1 & 0.97683 & 1 \\
\hline 15 & 0.99475 & 1 & 0.9896 & 1 & 0.98456 & 1 & 0.97963 & 1 \\
\hline 16 & 0.99538 & 1 & 0.99084 & 1 & 0.98639 & 1 & 0.98201 & 1 \\
\hline 17 & 0.99592 & 1 & 0.9919 & 1 & 0.98795 & 1 & 0.98406 & 1 \\
\hline 18 & 0.99638 & 1 & 0.99282 & 1 & 0.9893 & 1 & 0.98583 & 1 \\
\hline 19 & 0.99678 & 1 & 0.9936 & 1 & 0.99047 & 1 & 0.98737 & 1 \\
\hline 20 & 0.99713 & 1 & 0.99429 & 1 & 0.99148 & 1 & 0.98871 & 1 \\
\hline
\end{tabular}

From the table it can be observed that as the value of $\alpha$ increase the tail of the distribution becomes thicker.

A. Azzalini [10] introduced a class of skewed distributions with probability density function $2 f(x) F(\lambda x)$. N. Balakrishnan, R.S.Ambagaspitiya [11] defined a skew-Laplace distribution in line with [10]. T.J. Kuzubowski, K. Podogorski [12] studied asymmetric Laplace distribution with probability density function

$$
f(x ; k, \sigma)=\frac{1}{\sigma} \frac{k}{1+k^{2}}\left\{\begin{array}{ll}
e^{-\left(\frac{k}{\sigma}\right) x} & \text { if } x \geq 0 \\
e^{-\left(\frac{1}{k \sigma}\right) x} & \text { if } x<0
\end{array} \text { where } k>0\right.
$$

The characteristic function of asymmetric Laplace distribution with probability density function (3.5) is given by

$$
\varphi(t)=\frac{1}{1+(\sigma t)^{2}-i \mu t}
$$

where $\frac{1}{k}-k=\frac{\mu}{\sigma}$

The skew extension of GSDW distribution named as Generalized Asymmetric Double Weibull (GADW) distribution is defined as 


$$
g(x)= \begin{cases}\frac{k^{2}}{1+k^{2}} \frac{\alpha \beta}{\sigma} \frac{\left(\frac{k(x-\mu)}{\sigma}\right)^{\beta-1} e^{\left(\frac{k(x-\mu)}{\sigma}\right)^{\beta}}}{\left(e^{\left(\frac{k(x-\mu)}{\sigma}\right)^{\beta}}-\bar{\alpha}\right)^{2}}, x \geq \mu \\ \frac{1}{1+k^{2}} \frac{\alpha \beta}{\sigma} \frac{\left(\frac{-(x-\mu)}{k \sigma}\right)^{\beta-1} e^{\left(\frac{-(x-\mu)}{k \sigma}\right)^{\beta}}}{\left(e^{\left(\frac{-(x-\mu)}{k \sigma}\right)^{\beta}}-\bar{\alpha}\right)^{2}}, x<\mu\end{cases}
$$

The distribution function with the location parameter $\mu$ is

$$
F(x)= \begin{cases}\frac{k^{2}}{1+k^{2}} \frac{\alpha}{\left(e^{\left(\frac{-(x-\mu)}{k \sigma}\right)^{\beta}-\bar{\alpha}}\right)}, x<\mu \\ 1-\frac{1}{1+k^{2}} \frac{\alpha}{\left(e^{\left(\frac{k(x-\mu)}{\sigma}\right)^{\beta}}-\bar{\alpha}\right)}, x \geq \mu\end{cases}
$$

\section{Application}

In this section, we analyse the daily share index of Bombay stock exchange (INDIA) and find that among the available distributions the generalized asymmetric double Weibull distribution is the most suitable one.

The distribution of stock returns, commodity prices, foreign currency rates and other financial data have attracted the attention of numerous researchers. The first step towards the statistical modelling of stock price change was based on three assumptions: independence, identical distribution and finite variance of daily changes. The family of stable distributions seemed appropriate because they could allow independent and identically distributed returns and at the same time allow for the observed leptokurtic in the data. For the graphical methods of data analysis, see [13]

As a consequence of empirical evidence and fact that normal distribution is a special case of stable law, the stable assumption has enjoyed some popularity among financial modellers. However a number of empirical studies have provided evidence that it is consistent with the stable hypothesis, see $[14,15]$ and $[16]$. In response to these empirical inconsistencies alternative to the stable laws have been proposed for asset returns models. W. DuMouchel [17], investigates a mixture of normal and stable distributions. A. Boness et al.[18] proposes a mixture of normal distribution.

S. Mittnik, S.T. Rachev $[19,20]$ have considered various probability schemes and extended the stability concept of Mandelbrot, which arises from one specific summation scheme. These leads to a variety of distributions, stable with respect to underlying scheme. They also fitted these alternative stable distributions to stock index data and compared their appropriateness. Their findings were that the double Weibull distribution, which arises in the geometric summation scheme, dominates all other alternative stable laws.

The daily share index of Bombay stock exchange (INDIA) is considered. It is found that the most suitable distribution for modelling it is the generalized asymmetric double Weibull distribution. For modelling the data we have considered standard normal distribution, Laplace distribution, Asymmetric Laplace distribution, Asymmetric Linnik distribution, Cauchy distribution, Double Weibull distribution, generalized double Weibull distribution and generalized asymmetric double Weibull distribution.

The daily share index of Bombay stock exchange (INDIA from $1^{\text {st }}$ July 1997 to $10^{\text {th }}$ march 2008 (2649 observations) are considered for analysis. Logarithmic difference is taken and the resulting series is standardised and plotted in Fig.2. 


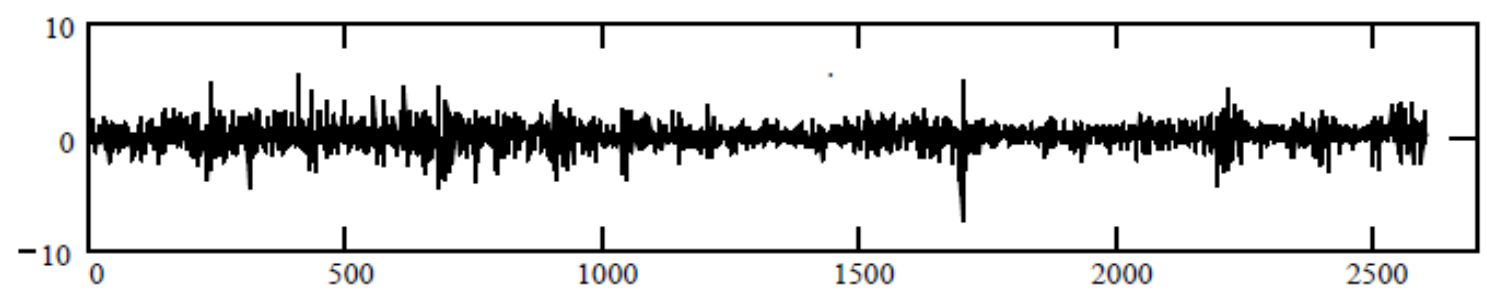

Fig.2.Error plot of the daily share index (BSE) INDIA (standardised)

The data exhibit some random nature and we are interested in modelling this nature. The maximum of observation is 5.318 , minimum is -7.399 and median is 0.041 . The histogram is plotted with 50 classes of equal width and it is given in Fig.3.

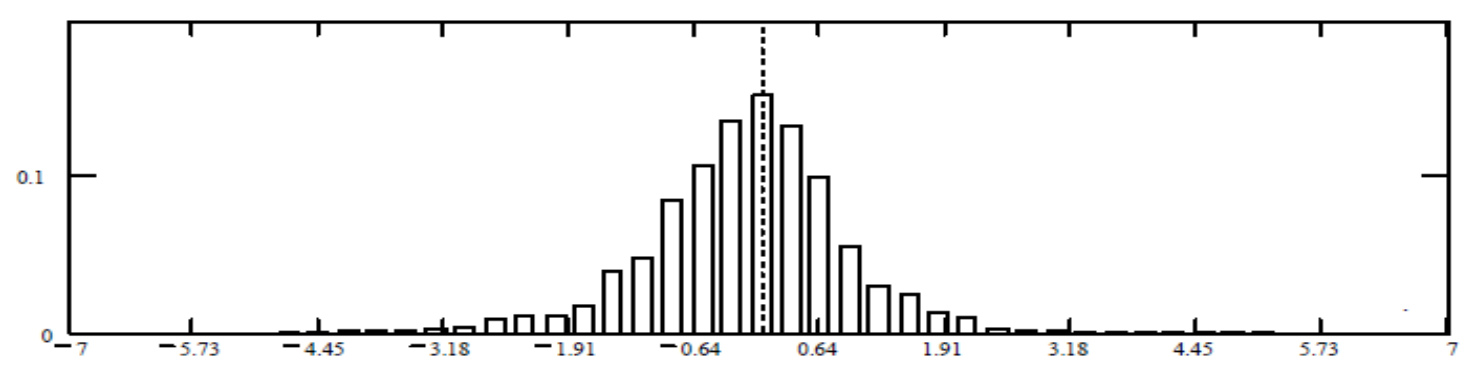

Fig.3.Histogram of the standardised error distribution of daily share index (BSE)

The data were fitted by various symmetric and asymmetric distributions and the comparisons are presented in Table 2.

Table 2.Table of critical values for Chi-square and Kolmogrov Smirnov test for various distributions

\begin{tabular}{|c|c|c|c|c|c|c|c|c|c|c|}
\hline Test & 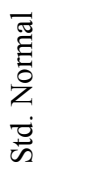 & $\begin{array}{l}.0 \\
.00 \\
000 \\
0 \\
1\end{array}$ & 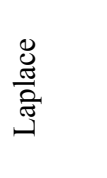 & 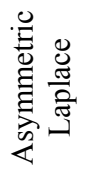 & 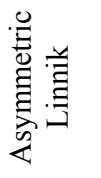 & $\begin{array}{l}\frac{\vec{e}}{0} \\
\frac{0}{\tilde{U}}\end{array}$ & 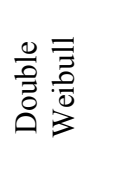 & 令 & 总 & : \\
\hline Chi-square & 294.18 & 64 & 75.9 & 72.6 & 98.18 & 278 & 60.7 & 44.87 & 40.404 & 44 \\
\hline $\begin{array}{c}\text { Kolmogrov } \\
\text { Smirnov }\end{array}$ & 0.045 & 0.03 & 0.026 & 0.036 & 0.035 & 0.096 & 0.019 & 0.023 & 0.02 & 0.032 \\
\hline
\end{tabular}

From the table it may be noted that the GADW distribution is far better in modelling the data than the other distributions considered. 2649 data are simulated from the fitted distribution and plotted in Figure 4 with the observed data (solid line)

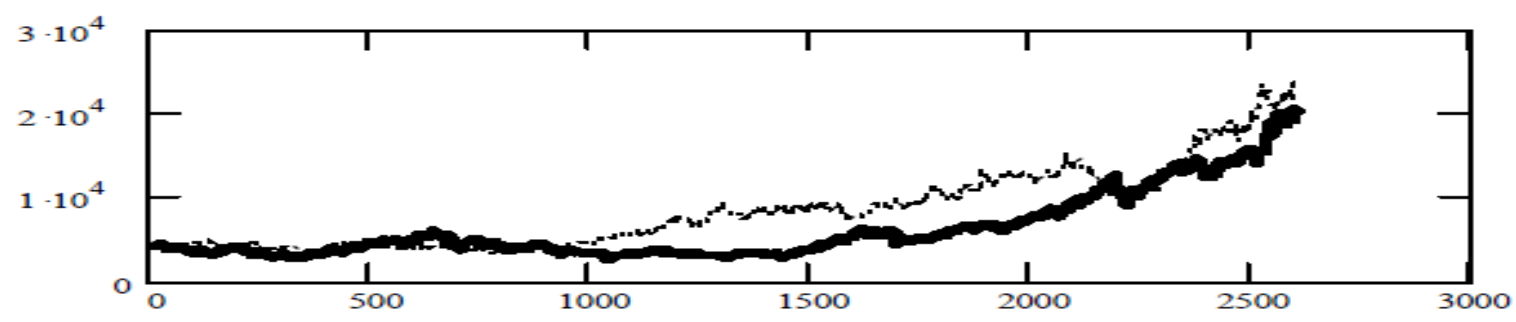

Fig.4.Data series and simulated series plotted together 
Table 3. Table of prediction for five days and observed data at those days

\begin{tabular}{llllll}
\hline Date & $28 / 12 / 2007$ & $31 / 12 / 2007$ & $02 / 01 / 2008$ & $03 / 01 / 2008$ & $04 / 01 / 2008$ \\
\hline Observed data & 20286.99 & 20465.3 & 20345.2 & 20686.89 & 20812.65 \\
Forecast & 20286.99 & 20238.71 & 20381.57 & 20468.85 & 20202.68 \\
\hline
\end{tabular}
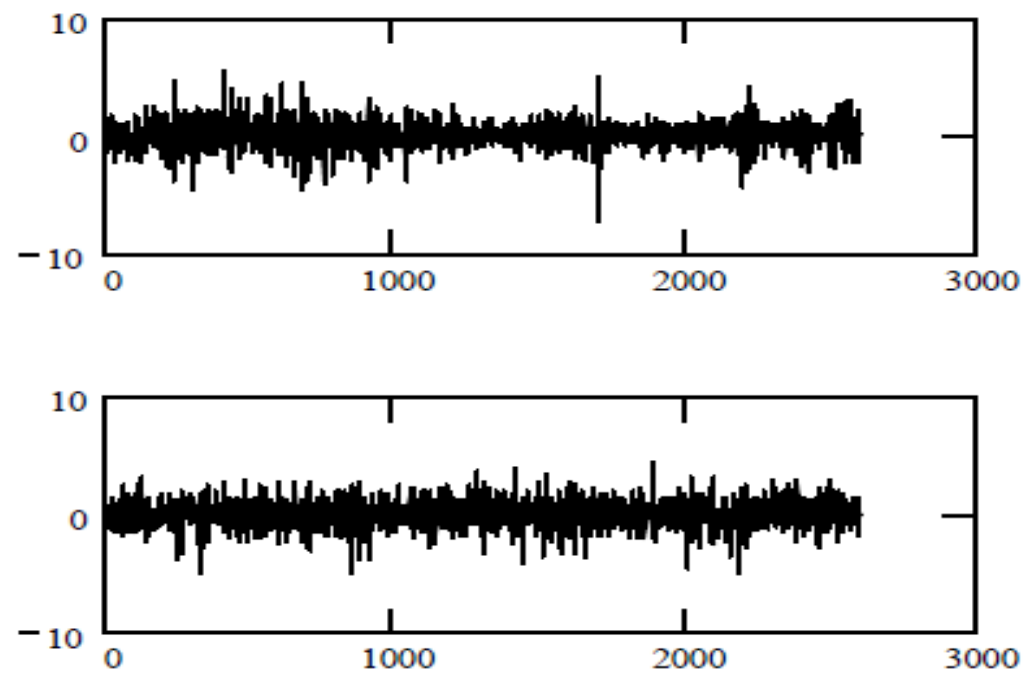

Fig.5.Error series plot of the observed data (up) and simulated data (down)

\section{References}

[1] N.L. Johnson, S. Kotz and N. Balakrishnan, Continuous Univariate Distributions, Volume 1, 2nd edn, (John Wiley \& Sons, Inc., New York., 1994).

[2] D.N.P. Murthy, M. Xie and R. Jiang, Weibull Models (Wiley, New Jersey, 2004).

[3] A.W. Marshall and I. Olkin, A new method for adding parameter to a family of distributions with application to exponential and Weibull families. Biometrika,84(1997)641-652.

[4]. L.V. Tavares, An exponential Markovian stationary process, Journal of Applied Probability 17(1980)1117 -1120.

[5] C.H. Sim, Simulation of Weibull and gamma autoregressive stationary process. Communications in Statistics Simulation and Computation 15(1986)1141 - 1146.

[6] K.K. Jose, S.R. Naik and M.M. Ristic, Marshall-Olkin q-Weibull distribution and max-min processes. Statist. Papers 51(2010)837-851.

[7] K.K. Jose, Marshall - Olkin Family of Distributions and their applications in reliability theory, time series modelling and stress - strength analysis. Int. Statistical Inst. Proc. $58^{\text {th }}$ World Statistical Congress, Dublin (Session CPS005), (2011) $3918-3923$

[8] A.M. Kagan,Yu. V. Linnik and C.R. Rao, Characterization Problems in Mathematical Statistics,(Wiley, New York. 1973).

[9] N. Balakrishnan and S. Kocherlakota, On the double Weibull distribution: order statistics and estimation, Sankhya, Series $B$ 47(1985)161-178.

[10] A. Azzalini, A class of distributions which includes the normal ones, Scandinanian Journal of Statistics 12(1985)171 - 178.

[11] N. Balakrishnan and R.S. Ambagaspitiya, On skewed Laplace distributions, (Report, McMaster University, Hamilton, Ontario, Canada,1994).

[12] T.J. Kozubowski and K. Podgorski, Asymmetric Laplace distributions. Mathematical Scientist 25(2000)37 - 46.

[13] J.M. Chambers, W.S. Cleveland, B. Kleiner and P.A. Tukey, Graphical methods for data analysis. Wadsworth. Belmont, C.A, (1983).

[14] R.R. Officer, The distribution of stock returns. Journal of the American Statistical Association 76(1972)807-812

[15] R.C. Blattberg and N.J.Gonedes, A comparison of the stable and student distribution as statistical model for stock prices, Journal of Business 47(1974)244 - 280.

[16] V. Akgiray and G.G. Booth, The stable law model of stock returns, Journal of the American Statistical Association 68(1988)469 $-477$.

[17] W. DuMouchel, Stable distributions in statistical inference: I symmetric stable distributions compared to other symmetric logtailed distributions. Journal of the American Statistical Association, 68(1973) 469 - 477.

[18] A. Boness, A. Chen and S. Jatusipatak, Investigations of non-stationary prices. Journal of Business 47 (1974)518 - 537.

[19] S. Mittnik and S.T. Rachev, Stable distributions for asset returns. Applied Mathematics Letters. 2(1989)301 - 304.

[20] S. Mittnik and S.T. Rachev,. Modelling asset returns with alternative stable laws. Economic Review 12(1993) 261 - 330. 